ELECTRONIC RESEARCH ANNOUNCEMENTS OF THE AMERICAN MATHEMATICAL SOCIETY

Volume 6, Pages 40-44 (June 6, 2000)

S $1079-6762(00) 00078-0$

\title{
MINIMAL VARIETIES OF ALGEBRAS OF EXPONENTIAL GROWTH
}

\author{
A. GIAMBRUNO AND M. ZAICEV
}

(Communicated by Efim Zelmanov)

\begin{abstract}
The exponent of a variety of algebras over a field of characteristic zero has been recently proved to be an integer. Through this scale we can now classify all minimal varieties of a given exponent and of finite basic rank. As a consequence we describe the corresponding T-ideals of the free algebra, and we compute the asymptotics of the related codimension sequences. We then verify in this setting some known conjectures.
\end{abstract}

\section{INTRODUCTION}

In this note we deal with the classification of varieties of associative algebras over a field of characteristic zero. A natural scale is provided by the so-called exponent of a variety, which has been recently proved to be an integer.

We recall the terminology and the basic definitions. Throughout, $F$ will be a field of characteristic zero, $X=\left\{x_{1}, x_{2}, \ldots\right\}$ a countable set and $F\langle X\rangle$ the free algebra on $X$ over $F$. Recall that a polynomial $f\left(x_{1}, \ldots, x_{n}\right) \in F\langle X\rangle$ is an identity for an associative $F$-algebra $A$ if $f\left(a_{1}, \ldots, a_{n}\right)=0$ for all $a_{1}, \ldots, a_{n} \in A$. The set of all polynomial identities of $A$ is a T-ideal $\operatorname{Id}(A)$ of $F\langle X\rangle$, i.e., an ideal invariant under all endomorphisms of $F\langle X\rangle$, and it is easy to see that all T-ideals of $F\langle X\rangle$ are of this type.

In order to study the properties shared by all algebras with a given set of polynomial identities or the T-ideals of $F\langle X\rangle$, the language of varieties seems the most appropriate. Recall that the class $\mathcal{V}$ of all $F$-algebras satisfying all the polynomial identities of a T-ideal $I$ is called the variety associated to $I$. We write $I=\operatorname{Id}(\mathcal{V})$ and, in case $\mathcal{V}$ is generated by an algebra $A$, we write $\mathcal{V}=\operatorname{var}(A)$. It is clear that $F\langle X\rangle / \operatorname{Id}(\mathcal{V})$ belongs to the variety $\mathcal{V}$ and is the relatively free algebra of countable rank of $\mathcal{V}$

An important invariant of a variety $\mathcal{V}$ is the so-called growth function or codimension growth, which we now describe: for every $n \geq 1$ let $P_{n}$ be the space of all multilinear polynomials in the variables $x_{1}, \ldots, x_{n}$. Since char $F=0$, by the well-known multilinearization or polarization process, a T-ideal $\operatorname{Id}(\mathcal{V})$ is determined by the multilinear polynomials it contains; hence the relatively free algebra $F\langle X\rangle / \operatorname{Id}(\mathcal{V})$ is determined by the sequence of subspaces $\left\{P_{n} /\left(P_{n} \cap \operatorname{Id}(\mathcal{V})\right)\right\}_{n \geq 1}$.

Received by the editors October 4, 1999.

2000 Mathematics Subject Classification. Primary 16R10, 16 P90.

Key words and phrases. Varieties of algebras, polynomial identities.

The first author was partially supported by MURST of Italy; the second author was partially supported by the RFBR grants 99-01-00233 and 96-15-96050. 
The integer $c_{n}(\mathcal{V})=\operatorname{dim} P_{n} /\left(P_{n} \cap \operatorname{Id}(\mathcal{V})\right)$ is called the $n$th codimension of $\mathcal{V}$, and the growth function determined by the integral sequence $\left\{c_{n}(\mathcal{V})\right\}_{n \geq 1}$ is the growth of the variety $\mathcal{V}$.

It is well known that if $\mathcal{V}$ is a nontrivial variety, then the sequence of codimensions is exponentially bounded ([14]), i.e., there exist constants $\alpha, a>0$ such that $c_{n}(\mathcal{V}) \leq$ $\alpha a^{n}$ for all $n$. Kemer in 10 characterized the varieties with a polynomially bounded codimension sequence (see also [9]). From his description it follows that there exists no variety with intermediate growth of the codimensions between polynomial and exponential.

To capture the exponential behavior of this sequence one defines $\overline{\operatorname{Exp}(\mathcal{V})}=$ $\limsup _{n \rightarrow \infty} \sqrt[n]{c_{n}(\mathcal{V})}, \quad \underline{\operatorname{Exp}(\mathcal{V})}=\liminf _{n \rightarrow \infty} \sqrt[n]{c_{n}(\mathcal{V})}$ and, in the case of equality, $\operatorname{Exp}(\mathcal{V})=\overline{\operatorname{Exp}(\mathcal{V})}=\underline{\operatorname{Exp}(\mathcal{V})}$. In the 80's it was conjectured by Amitsur that for any nonnilpotent proper variety $\mathcal{V}, \operatorname{Exp}(\mathcal{V})$ exists and is an integer. This conjecture has been recently verified. For a nilpotent variety $\mathcal{V}$, define $\operatorname{Exp}(\mathcal{V})=0$. Then we have

Theorem ([7], [8]). For any proper variety $\mathcal{V}, \operatorname{Exp}(\mathcal{V})$ exists and is an integer.

The integer $\operatorname{Exp}(\mathcal{V})$ is called the exponent of the variety $\mathcal{V}$.

Among varieties of a given exponent a prominent role is played by the so-called minimal varieties. A variety of exponent $d$ is minimal if every proper subvariety has exponent strictly less than $d$. From Kemer's result [10] it turns out that the infinite dimensional Grassmann algebra $G$ and the algebra of $2 \times 2$ upper triangular matrices $U T_{2}$ generate the only two minimal varieties of exponent 2. Moreover, Drensky in [4] and [5] has proved that any polynomial of the type $\left[x_{1}, x_{2}\right] \cdots\left[x_{2 n-1}, x_{2 n}\right]$ or $\left[x_{1}, x_{2}, x_{3}\right] \cdots\left[x_{3 n-2}, x_{3 n-1}, x_{3 n}\right]$ determines a minimal variety.

Here we shall classify all minimal varieties of finite basic rank. Recall that a variety $\mathcal{V}$ has finite basic rank if $\mathcal{V}=\operatorname{var}(A)$, where $A$ is a finitely generated algebra. According to a result of Kemer such a variety can be generated by a finite dimensional algebra; another equivalent formulation is that $\mathcal{V}$ satisfies all Capelli polynomials of some rank (see [11).

We shall describe the minimal varieties of finite basic rank in terms of generating algebras and in terms of identities. As a consequence we shall verify for these varieties a conjecture of Drensky stating that a variety $\mathcal{V}$ is minimal if and only if $\operatorname{Id}(\mathcal{V})$ is a product of so-called verbally prime T-ideals.

As a corollary we shall also find the exact asymptotics for the codimension sequence of such minimal varieties. Recall that two functions of a natural variable $f(n)$ and $g(n)$ are asymptotically equal and we write $f(n) \simeq g(n)$, if $\lim _{n \rightarrow \infty} \frac{f(n)}{g(n)}=$ 1.

Regev and Berele ([15], [2]) computed the asymptotics for some important classes of algebras, in particular for the matrix algebras over $F$. In all cases they found that

$$
c_{n}(A) \simeq \alpha n^{g} d^{n},
$$

where $d \geq 1$ is an integer, $g$ is a half-integer and $\alpha$ is a constant. Generalizing Amitsur's conjecture Regev formulated the hypothesis that a relation of type (1.1) holds for any algebra $A$ satisfying a nontrivial identity (PI-algebra). Our description of minimal varieties together with results of Berele, Regev and Lewin allows us to explicitly compute the asymptotics of $c_{n}(A)$ for any upper block-triangular matrix 
algebra over $F$. This confirms Regev's conjecture for any minimal variety of finite basic rank.

Finally we discuss the relationship between growth of algebras and growth of identities. We find some relations between the exponent of the codimension growth and the Gelfand-Kirillov dimension of a finitely generated relatively free algebra.

\section{UPPER BLOCK-TRIANGULAR MATRIX ALGEBRAS}

Let $d_{1}, \ldots, d_{m}$ be positive integers and let $U T\left(d_{1}, \ldots, d_{m}\right)$ denote the algebra of all upper block-triangular matrices over $F$ of the type

$$
\left(\begin{array}{cccc}
M_{d_{1}}(F) & & & * \\
0 & \ddots & & \\
\vdots & & & \\
0 & \cdots & 0 & M_{d_{m}}(F)
\end{array}\right)
$$

where $M_{d_{i}}(F)$ is the algebra of $d_{i} \times d_{i}$ matrices over $F$.

The Wedderburn-Malcev decomposition of this algebra is readily written as

$$
U T\left(d_{1}, \ldots, d_{m}\right) \cong M_{d_{1}}(F) \oplus \cdots \oplus M_{d_{m}}(F)+J,
$$

where $J$ is the Jacobson radical of $U T\left(d_{1}, \ldots, d_{m}\right)$ and it consists of all strictly upper block-triangular matrices. Invoking [7] it is easy to see that

$$
\operatorname{Exp}\left(U T\left(d_{1}, \ldots, d_{m}\right)\right)=d_{1}^{2}+\cdots+d_{m}^{2} .
$$

The next result shows that algebras of upper block-triangular matrices arise naturally in any finite dimensional algebra.

Lemma 2.1. Let $F$ be an algebraically closed field of characteristic zero. If $A$ is a finite dimensional algebra over $F$ and $\operatorname{Exp}(A)=d \geq 2$, then there exists a subalgebra of $A$ isomorphic to $U T\left(d_{1}, \ldots, d_{m}\right)$ with $d_{1}^{2}+\cdots+d_{m}^{2}=d$.

What about comparing the identities of two algebras of upper block-triangular matrices? Let $A=U T\left(d_{1}, \ldots, d_{m}\right)$ and $B=U T\left(q_{1}, \ldots, q_{s}\right)$ be two such algebras and suppose that $q=q_{1}+\cdots+q_{s} \leq d=d_{1}+\cdots+d_{m}$. Then one can consider the canonical embeddings of $A$ and $B$ into $M_{d}(F)$. In this case $B$ lies inside $A$ if and only if for any $j \in\{1, \ldots, m\}$ there exists $i \in\{1, \ldots, s\}$ such that $q_{1}+\cdots+q_{i}=$ $d_{1}+\cdots+d_{j}$ provided that $d_{1}+\cdots+d_{j} \leq q$. It turns out that $B$ satisfies all identities of $A$ if and only if $B$ is a subalgebra of $A$.

Lemma 2.2. Let $A=U T\left(d_{1}, \ldots, d_{m}\right)$ and $B=U T\left(q_{1}, \ldots, q_{s}\right)$ be two upper blocktriangular matrix algebras over $F$. Then $B \in \operatorname{var}(A)$ if and only if $B \subseteq A$.

It is possible to compute the T-ideal of identities of an upper block-triangular matrix algebra. The main tool for this proof is a result of Lewin [12]; it turns out that such a T-ideal is a product of T-ideals of identities of matrix algebras.

Theorem 2.3. The T-ideal of identities of the algebra of upper block-triangular matrices $U T\left(d_{1}, \ldots, d_{m}\right)$ is

$$
\operatorname{Id}\left(U T\left(d_{1}, \ldots, d_{m}\right)\right)=\operatorname{Id}\left(M_{d_{1}}(F)\right) \cdots \operatorname{Id}\left(M_{d_{m}}(F)\right) .
$$




\section{Minimal VARIETIES OF FINITE BASIC RANK}

The algebras of upper block-triangular matrices provide generators for the minimal varieties of finite basic rank. In fact we can prove

Theorem 3.1. Let $\mathcal{V}$ be a variety of finite basic rank and suppose $\operatorname{Exp}(\mathcal{V})=d \geq 2$. Then $\mathcal{V}$ is a minimal variety of exponent $d$ if and only if $\mathcal{V}$ is generated by some upper block-triangular matrix algebra $U T\left(d_{1}, \ldots, d_{m}\right)$ with $d_{1}^{2}+\cdots+d_{m}^{2}=d$.

Theorem 3.1 implies the following interesting remarks:

Corollary 1. For any positive integer $k$ there exists a variety $\mathcal{V}$ such that $\operatorname{Exp}(\mathcal{V})=$ $d$ but $\operatorname{Exp}(\mathcal{U}) \leq d-k$ for any proper subvariety $\mathcal{U} \subset \mathcal{V}$.

Corollary 2. For any integer $d \geq 2$, the number of minimal varieties of finite basic rank and of exponent $d$ is finite.

Kemer introduced the notion of verbally prime T-ideal. Recall that $I$ is such a T-ideal if $f g \in I$ implies that either $f \in I$ or $g \in I$, where $f$ and $g$ are two polynomials in $F\langle X\rangle$ in disjoint sets of variables. It turns out that for a finitely generated algebra $A, \operatorname{Id}(A)$ is verbally prime if and only if $\operatorname{Id}(A)=\operatorname{Id}\left(M_{d}(F)\right)$ for some $d \geq 1$.

Drensky in [4] conjectured that a variety $\mathcal{V}$ is minimal if and only if $\operatorname{Id}(\mathcal{V})$ is a product of verbally prime T-ideals. As a consequence of Theorem 2.3 we are now able to confirm this conjecture for varieties of finite basic rank.

Corollary 3. Let $\mathcal{V}$ be a variety of finite basic rank and let $I=I d(\mathcal{V})$. Then $\mathcal{V}$ is minimal of exponent $d \geq 2$ if and only if $I=I_{1} \cdots I_{k}$, where $I_{1}, \ldots, I_{k}$ are verbally prime T-ideals.

Another application of Theorem 2.3 is a precise asymptotic formula for the codimension growth of upper block-triangular matrix algebras. From [15] and [3] Theorem 1.4] immediately follows

Theorem 3.2. Let $A=U T\left(d_{1}, \ldots, d_{m}\right)$. Then the asymptotic behavior of its codimensions is described by the formula

$$
c_{n}(A) \simeq \alpha n^{g} d^{n}
$$

with $d=d_{1}^{2}+\cdots+d_{m}^{2}, g=-\frac{1}{2}\left(\sum_{i=1}^{m} d_{i}^{2}-3 m+2\right)$ and

$$
\alpha=\alpha_{1} \cdots \alpha_{m} \frac{d_{1}^{g_{1}} \cdots d_{m}^{g_{m}}}{\left(d_{1}^{2}+\cdots+d_{m}^{2}\right)^{g}}
$$

where $g_{i}=-\left(d_{i}^{2}-1\right)$,

$$
\alpha_{i}=\left(\frac{1}{\sqrt{2 \pi}}\right)^{d_{i}-1} \cdot\left(\frac{1}{2}\right)^{\frac{1}{2}\left(d_{i}^{2}-1\right)} \cdot 1 ! \cdot 2 ! \cdots\left(d_{i}-1\right) ! d_{i}^{\frac{1}{2}\left(d_{i}^{2}+4\right)}
$$

Hence the previous theorem confirms Regev's conjecture for minimal varieties of finite basic rank.

The Gelfand-Kirillov dimension of PI-algebras has been extensively investigated in the last years (see, for example, [6]). We point out some relations between such dimension of a relatively free algebra and the codimension growth of the corresponding variety (see also [13] and [1]). For a finitely generated algebra $A$ we denote by GKdim $A$ the Gelfand-Kirillov dimension of $A$. 
Theorem 3.3. Let $\mathcal{V}$ be a nontrivial variety and $R_{m}=R_{m}(\mathcal{V})$ the relatively free $\mathcal{V}$-algebra with $m$ generators. Then

$$
\operatorname{GKdim} R_{m} \geq(m-1) \operatorname{Exp}\left(R_{m}\right)+1 \text {. }
$$

If $\mathcal{V}=\operatorname{var} U T\left(d_{1}, \ldots, d_{k}\right)$ and $m \geq 2$, then

$$
\operatorname{GKdim} R_{m}=(m-1) \operatorname{Exp}\left(R_{m}\right)+k=(m-1)\left(d_{1}^{2}+\cdots+d_{k}^{2}\right)+k .
$$

\section{REFERENCES}

1. A. Berele, Generic verbally prime PI-algebras and their GK-dimension, Comm. Algebra 21 (1993), 1478-1504. MR 94f:16038

2. A. Berele and A. Regev, On the codimensions of the verbally prime P.I. algebras, Israel J. Math. 91 (1995), 239-247. MR 96g:16028

3. A. Berele and A. Regev, Codimensions of products and intersections of verbally prime Tideals, Israel J. Math. 103 (1998), 17-28. MR 99b:16037

4. V. Drensky, Extremal varieties of algebras I, Serdica 13 (1987), 320-332. (Russian) MR 90e:08010

5. V. Drensky, Extremal varieties of algebras II, Serdica 14 (1988), 20-27. (Russian) MR 90e:08011

6. V. Drensky, Gelfand-Kirillov dimension of PI-algebras, In: Methods in Ring Theory, Lect. Notes in Pure and Appl. Math., vol. 198, Marcel Dekker, New York, 1998, pp. 97-113.

7. A. Giambruno and M. Zaicev On codimension growth of finitely generated associative algebras, Adv. Math. 140 (1998), 145-155. MR 99k:16049

8. A. Giambruno and M. Zaicev, Exponential codimension growth of PI-algebras: an exact estimate, Adv. Math. 142 (1999), 221-243. MR 2000a:16048

9. A. Giambruno and M. Zaicev, A characterization of algebras with polynomial growth of the codimensions, Proc. Amer. Math. Soc. (to appear).

10. A. Kemer, T-ideals with power growth of the codimensions are Specht, Sibirskii Matematicheskii Zhurnal, 19 (1978), 54-69; English translation: Siberian Math. J. 19 (1978), 37-48. MR 57:6070

11. A. Kemer, Ideals of identities of associative algebras, AMS Translations of Mathematical Monographs, Vol. 87, 1988. MR 92f:16031

12. J. Lewin, A matrix representation for associative algebras. I, Trans. Amer. Math. Soc. 188 (1974), 293-308. MR 49:2848

13. C. Procesi, Non-commutative affine rings, Atti Acc. Naz. Lincei, Ser. VIII 8 (1967), 239-255. MR 37:256

14. A. Regev, Existence of identities in $A \otimes B$, Israel J. Math. 11 (1972), 131-152. MR 47:3442

15. A. Regev, Codimensions and trace codimensions of matrices are asymptotically equal, Israel J. Math. 47 (1984), 246-250. MR 85j:16024 ITALY

Dipartimento di Matematica ed Applicazioni, Università di Palermo, 90123 Palermo,

E-mail address: a.giambruno@unipa.it

Department of Algebra, Faculty of Mathematics and Mechanics, Moscow State UniVERSiTy, Moscow 119899, Russia

E-mail address: zaicev@mech.math.msu.su 